\title{
Measurement of the lowest dosage of phenobarbital that can produce drug discrimination in rats
}

\author{
Donald A. Overton • Gregg D. Stanwood • \\ Bhavesh N. Patel • Sreenivasa R. Pragada • \\ M. Kathleen Gordon
}

Received: 20 May 2008 / Accepted: 22 November 2008 / Published online: 13 December 2008

(C) Springer-Verlag 2008

\begin{abstract}
Rationale Accurate measurement of the threshold dosage of phenobarbital that can produce drug discrimination (DD) may improve our understanding of the mechanisms and properties of such discrimination.

Objectives This study aimed to compare three methods for determining the threshold dosage for phenobarbital (D) versus no-drug (N) DD.

Materials and methods Rats learned a D versus N DD in two-lever operant training chambers. A titration scheme was employed to increase or decrease dosage at the end of each
\end{abstract}

D. A. Overton

Department of Psychology, Temple University,

Philadelphia, PA 19122, USA

\section{G. D. Stanwood}

Department of Pharmacology, School of Medicine,

Vanderbilt University,

Nashville, TN 37232, USA

B. N. Patel

Engineering Department, Walmart,

7000 Marina Blvd.,

Brisbane, CA 94005, USA

S. R. Pragada

ExeCue, Inc.,

1660 Crescent Dr.,

Tarrytown, NY 10591, USA

M. K. Gordon

Department of Psychology, University of Delaware,

Newark, DE 19716, USA

D. A. Overton $(\bowtie)$

605 Bobbin Mill Road,

Media, PA 19063, USA

e-mail: doverton@temple.edu 18-day block of sessions depending on whether the rat had achieved criterion accuracy during the sessions just completed. Three criterion rules were employed, all based on average percent drug lever responses during initial links of the last six $\mathrm{D}$ and six $\mathrm{N}$ sessions of a block. The criteria were: $\mathrm{D} \%>66$ and $\mathrm{N} \%<33 ; \mathrm{D} \%>50$, and $\mathrm{N} \%<$ $50 ;(\mathrm{D} \%-\mathrm{N} \%)>33$. Two squads of rats were trained, one immediately after the other.

Results All rats discriminated drug versus no drug. In most rats, dosage decreased to low levels and then oscillated near the minimum level required to maintain criterion performance. The lowest discriminated dosage significantly differed under the three criterion rules. The squad that was trained second may have benefited by partially duplicating the lever choices of the previous squad.

Conclusions The lowest discriminated dosage is influenced by the criterion of discriminative control that is employed and is higher than the absolute threshold at which discrimination entirely disappears. Threshold estimations closer to absolute threshold can be obtained when criteria are employed that are more permissive of errors and that allow rats to maintain lever preferences.

Keywords Drug discrimination · Phenobarbital ·

Threshold dosage

\section{Introduction}

Studies that determine the lowest dosage that can be discriminated in a drug discrimination (DD) paradigm have been used to answer various scientific questions (e.g., Preston and Bigelow 1998; Zenick and Goldsmith 1981). The present study aimed to improve our understanding of the methods used to determine threshold for discriminative 
drug effects and to determine the lowest dosage of phenobarbital that can control drug discrimination.

Previous studies seeking to determine the lowest dose that can maintain discriminative control in animals have used at least three types of procedures: (1) train several groups of subjects to discriminate various doses to determine the lowest dose that can be discriminated (Colpaert et al. 1980b; Overton 1982); (2) train a single group to discriminate progressively reduced doses using a fixed regimen that incrementally reduces dose until the discrimination is lost (Colpaert et al. 1980a); (3) train a single group to discriminate progressively reduced doses using a titration procedure in which dosage is decreased until discrimination is lost, then increased till it reappears, decreased till it disappears, etc. (Overton 1979). If rats can discriminate lower doses when a fading regimen is employed, then the second paradigm takes advantage of that fact. The third procedure allows fading and gives the animal repeated opportunities to discriminate low doses instead of imposing an experimenter-specified schedule of dosage reductions. A priori, one might expect the titration procedure to yield the lowest thresholds since the animal has an essentially unlimited amount of time to learn to discriminate the near-threshold dosages. Analogous titration procedures have been employed with exteroceptive sensory stimuli (e.g., Blough and Blough 1977).

It appears to us that DD titration studies do not identify the absolute threshold below which no discriminative control occurs but instead identify a somewhat higher dosage at which response control is sufficient to meet or exceed the criterion. This lowest discriminated dosage (LD) presumably is influenced by the stringency of the criterion for discrimination that is adopted.

To clarify the characteristics of DD titration procedures, the present experiment tested the effect of variations in the criterion for discriminative control on the resulting LD.

\section{Materials and methods}

Subjects Twenty-four adult Long Evans male rats each weighing 320 to $465 \mathrm{~g}$ were purchased from Blue Spruce Farms (Altamont, NY, USA), quarantined, accustomed to a 23-h water deprivation schedule, shaped to lever press for water reinforcement under a fixed ratio 20 (FR-20) schedule in single-lever operant chambers, and then entered into drug versus no-drug DD training in two-lever chambers. Food was available at all times in the home cages. Water was available for $30 \mathrm{~min}$ daily starting $30 \mathrm{~min}$ after the training session. A diurnal $12 \mathrm{~h} / 12 \mathrm{~h}$ light/dark cycle was employed with sessions conducted near the middle of the light cycle. All experiments were approved by the Temple University Animal Care and Use Committee.
Apparatus DD training took place in Gerbrands Convertacage operant conditioning chambers $30 \times 32 \times 30 \mathrm{~cm}$ high. Each chamber had a Lucite door, aluminum walls and ceiling, and a steel-rod floor. Two levers mounted $19 \mathrm{~cm}$ apart were centered on the rear wall $3 \mathrm{~cm}$ above the floor. Between the levers, a water spout positioned $2 \mathrm{~cm}$ above the floor allowed delivery of $0.1 \mathrm{ml}$ of distilled water as reinforcer. Two $3-\mathrm{W}$ light bulbs mounted in the ceiling were illuminated when contingent reinforcement was available. The entire chamber was enclosed in a light-proof sound-attenuating enclosure with a ventilation fan providing a low level of white noise. Lever pressing was monitored, and house lights and reinforcement were controlled by Med. Associates, Inc. interface equipment. Data recording and contingency control were performed by MED-PC state notation software (Tatham and Zurn 1989).

Drugs Isotonic saline (N) or phenobarbital (D) was injected intraperitoneally $20 \mathrm{~min}$ before each session. Sodium phenobarbital dosages (including the weight of the Na salt) were $30,24,19,15.5,12.5,10,8,6,5,4,3,2.5,2,1.5$, $1.25,1.0,0.8$, or $0.6 \mathrm{mg} / \mathrm{kg}$. Dosage was $30 \mathrm{mg} / \mathrm{kg}$ during the first block of sessions. Thereafter, dosage of phenobarbital was titrated during successive blocks of sessions using a procedure in which dosage was decreased until accuracy of discrimination dropped below criterion, then increased until criterion accuracy reappeared, decreased till it disappeared, etc.

Training sessions Each daily training session consisted of six "minisessions" separated from one another by 30 -s time-outs during which house lights were turned off. Each minisession lasted until six reinforcers were earned or for a maximum of 4 min. Training was conducted 6 or 7 days/week.

Schedules of reinforcement During each session, one lever was designated as correct and the other as incorrect, with opposite lever assignments employed during $\mathrm{D}$ and $\mathrm{N}$ sessions. For all rats, lever 1 was correct during D sessions and lever 2 during $\mathrm{N}$ sessions. Only responding on the correct lever was reinforced. Each minisession consisted of an initial link (IL) followed by a terminal link (TL). Each IL lasted until ten presses had occurred either on lever 1 or on lever 2; a water reinforcer was delivered when the IL terminated only if the FR-10 was completed on the correct lever. During TLs of each minisession, an interlocked FR10/FI-90-s schedule was in effect on the correct lever. Under this schedule, one press was subtracted from the ratio requirement every $10 \mathrm{~s}$ until a minimum FR-1 requirement remained. Rates and temporal patterns of responding under the interlocked schedule approximate those seen with a simple FR-10 schedule, but it has the advantage that 
whenever drug substantially decreases rate of responding the ratio requirement is automatically reduced. Responses on the incorrect lever had no programmed consequence.

Discrimination training Rats were trained to discriminate between phenobarbital and saline. Training consisted of 378 sessions subdivided into 21 blocks, each of 18 sessions. During each block, the sequence of $\mathrm{D}$ and $\mathrm{N}$ sessions was DDNDNNDNDNDNDNDNDN. The irregular sequence during the first six sessions (DDNDNN) was used to reduce the likelihood that rats would learn to alternate their lever selection during successive sessions. Performance during the first initial link of the subsequent 12 sessions, during which $\mathrm{D}$ and $\mathrm{N}$ conditions alternated, was used to determine whether discriminative control was above or below criterion. The rats were assigned to two training squads, each of 12 rats. Squad 1 was always trained a few minutes before squad 2 and sessions and lever assignments were synchronized in both squads which made it possible, in principle, for rats in the second squad to improve their accuracy by duplicating the lever selection reinforced in squad 1. Due to a procedural error, data from squad 2 after training block \# 18 are not reported.

Criteria for dosage titration Criterion was specified in terms of the average percentage of presses on the drug lever during the first IL of the last 12 sessions of the block. The first IL of each session was used because behavior during that interval best differentiates a discriminating from a nondiscriminating rat. The last 12 sessions of each cycle were averaged as that gave the rats six sessions to acclimate to the newly imposed training dose before the criterion series of sessions began. In three groups, each of eight rats, the criteria were as follows.

Group 1: Criterion $=66 \%$ correct in both states. If (average percent $\mathrm{D}$-lever presses during six $\mathrm{D}$ sessions)> 66\% and (average percent $\mathrm{D}$-lever presses during six $\mathrm{N}$ sessions) $<33 \%$, then decrease dose $20 \%$, else increase dose $25 \%$ but not above $30 \mathrm{mg} / \mathrm{kg}$.

Group 2: Criterion $=50 \%$ correct in both states. Same titration rules as group 1, but using $50 \%$ as the criterion level of accuracy.

Group 3: Criterion $=33 \%$ difference between states. If (average percent D-lever presses during six D sessions) minus (average percent D-lever presses during six $\mathrm{N}$ sessions) $>33 \%$, then decrease dose $20 \%$, else increase dose $25 \%$ but not above $30 \mathrm{mg} / \mathrm{kg}$.

The criteria in groups 2 and 3 were more permissive than the criterion used in group 1. In group 2, accuracy of lever selection could be lower than in group 1 . In group 3, the criterion required the same percentage difference between $\mathrm{D}$ and $\mathrm{N}$ days as in group 1 but allowed a biased rat to meet criterion, e.g., $10 \%$ versus $45 \%$ D-lever choices during $\mathrm{N}$ and $\mathrm{D}$ sessions, respectively, would meet criterion only in group 3.

Hypotheses We predicted that more stringent criteria would lead to higher LD doses and less stringent criteria to lower LD doses and that rats in squad 2 might appear to discriminate lower dosages because they successfully copied the lever selections made by the rats in squad 1 a few minutes previously.

\section{Results}

Acquisition of discrimination Initial acquisition of the phenobarbital 30 versus no-drug discrimination was uneventful. Figure 1 shows the training dose during each block of sessions for each rat. Out of 24 rats, 21 met the criterion for discriminative control during the first 18-day block of training; the remainder met criterion during the second block. For the 21 rats, latency to begin pressing at the beginning of ILs reached asymptote within ten to 25 sessions, as did rate of responding and number of reinforcers earned per session; the remaining three rats required as many as 60 sessions before asymptotic responding was achieved.

Most rats showed a "bias" toward the no-drug lever, i.e., a tendency to select the no-drug lever during ILs somewhat more frequently than they chose the $\mathrm{D}$ lever. Average accuracy during ILs of blocks 4-9 (doses 15.5 to 5 in most rats) was $91.0 \%$ during $\mathrm{N}$ sessions and $81.4 \%$ during $\mathrm{D}$ sessions $(P<0.001$, analysis of variance (ANOVA)).

All rats dropped below criterion accuracy when training dose was sufficiently reduced. Thereafter, most rats regained criterion when dosage was increased by one or two steps, and subsequently the dose oscillated near threshold for most rats.

Fixations In five rats, a "fixation" (a strong persistent preference for one lever) appeared on one or more occasions. In these rats, once discriminative control was lost due to a dosage reduction, a small increase in dose did not immediately restore the discrimination. Instead, these rats sometimes failed to discriminate until dose had been raised to as high as four times the level at which the discrimination was lost.

Determination of lowest discriminated dose By inspection of the plots for dosage during successive blocks (Fig. 1), the LD was identified for each rat. This was defined as the lowest training dose that maintained above-criterion 

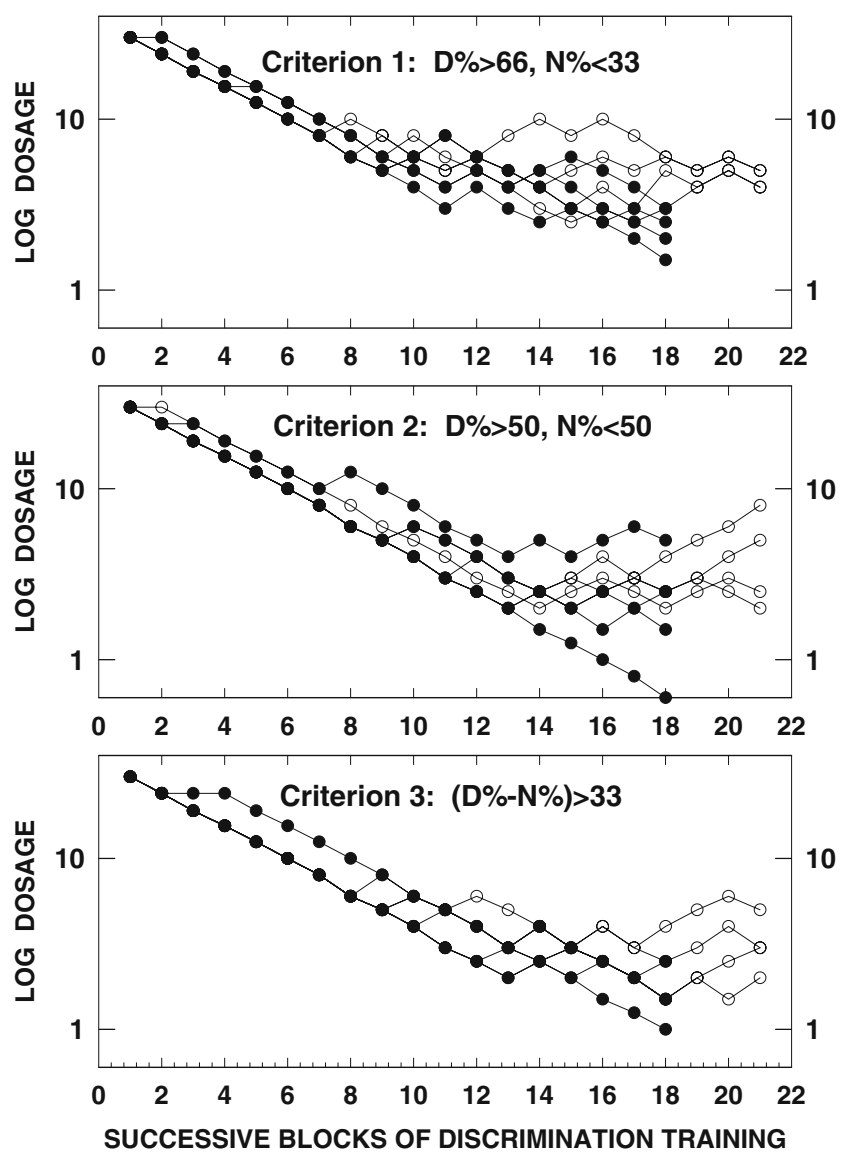

Fig. 1 Training dosage during successive 18-day blocks of training sessions for each rat. $Y$-axis is phenobarbital dosage in milligram per kilogram, ip. $X$-axis is successive 18 -day blocks. For all rats, training dose started at $30 \mathrm{mg} / \mathrm{kg}$ and during successive blocks was adjusted upward or downward using a titration algorithm based on accuracy of discrimination during the immediately preceding block of training sessions. The top, middle, and bottom panels show data for the rats trained with criteria 1,2 , and 3 , respectively. Open circles are rats in squad 1 and closed circles squad 2

performance during one or more phases of training. Column 4 in Table 1 lists the LD for each rat.

Other indices of discrimination The table also presents the average training dose during training blocks 13 through 18 and the percent correct presses during the first IL of blocks 13-18 computed by averaging IL accuracy across the last ten sessions of those blocks.

Statistical tests Two-factor ANOVAs were used to analyze performance during session blocks 13 through 18 (factor 1, criterion, three levels; factor 2, squad, two levels).

A two-factor ANOVA applied to LD values from Table 1 showed the difference between criteria 1 and 2 and between criteria 1 and 3 to be significant $(P<0.05$ and 0.02 , respectively). The difference between squads 1 and 2 was marginally significant $(P<0.10)$. A one-tailed test is permissible here as the a priori prediction is one-sided; squad 2 may have lower LD values than squad 1 but not the reverse.

ANOVA applied to the average training dose during blocks $13-18$ yielded $P<0.02$ and $P<0.02$ for the differences between criteria 1 versus 2 and 1 versus 3 . The difference between criteria 2 versus 3 and the difference between squads did not achieve significance.

ANOVA applied to the percent correct initial link responses averaged across sessions $7-16$ of training blocks 13-18 showed that criteria 1 versus 2 and 1 versus 3 differed $(P<0.01$ in both cases). The difference between criteria 2 and 3 and the effect of squad were not significant. In all ANOVAs, the interaction between squad and criterion did not approach significance.

Absolute threshold for discrimination LD is the lowest dosage that was discriminated at a criterion level of accuracy but is not the absolute threshold dose at which the amount of discriminative control reaches zero. Figure 2 illustrates this situation by showing average percent presses on the D lever as a function of relative training dosage. Plotting the results in this way slides the data for individual rats left or right so that performance at the LD dosage is plotted at the same location on the $X$-axis for each rat. The figure shows that rats exhibited a moderately high level of discriminative control at the LD (where relative dosage $=1$ ); at this dose, the average percent $\mathrm{D}$ presses was $68 \%$ during D sessions and $21 \%$ during $\mathrm{N}$ sessions. The figure suggests that zero amounts of discriminative control would occur only at a dose somewhat lower than LD, perhaps in the vicinity of $0.57 \times$ LD. Since the overall geometric mean $L D$ was $2.6 \mathrm{mg} / \mathrm{kg}$, then the average absolute threshold may have been approximately $1.5 \mathrm{mg} / \mathrm{kg}$.

\section{Discussion}

The discrimination was learned and the dosage titration procedure worked well in most rats. The predicted effects of criterion stringency on the lowest discriminated dose and on the average training dose during cycles 13-18 were statistically significant.

We had not expected rats in squad 2 to copy the lever choice of those in squad 1 and took no precautions to prevent this from happening. The results suggest that the rats in squad 2 were able to duplicate the lever choice of the previous rat to some small degree. This possibility was described by Colpaert et al (1977) and studied in some detail by Extance and Goudie (1981). The present study adds little to our understanding of this phenomenon.

The elevated percent correct responses in rats trained with criterion 1 agrees with expectations because that 
Table 1 Lowest discriminated dosage, average dosage, and average percent correct responses for each rat, criterion, and training squad

\begin{tabular}{lllll}
\hline Group Rat & $\begin{array}{l}\text { Training } \\
\text { squad }\end{array}$ & $\begin{array}{l}\text { Lowest } \\
\text { discriminated } \\
\text { dosage (LD) }\end{array}$ & $\begin{array}{l}\text { Average } \\
\text { dosage in } \\
\text { sessions } \\
13-18\end{array}$ & $\begin{array}{l}\text { Average percent } \\
\text { correct in } \\
\text { sessions 13-18 }\end{array}$ \\
& & &
\end{tabular}

\begin{tabular}{|c|c|c|c|c|}
\hline \multicolumn{5}{|c|}{ Criterion 1: $(\mathrm{D}$ IL\%) $>66$ and $(\mathrm{N}$ IL\%) $<3$} \\
\hline 1 & 1 & 1 & 6 & \\
\hline 1 & 2 & 1 & 3 & \\
\hline 1 & 3 & 1 & 5 & \\
\hline 1 & 4 & 1 & 4 & \\
\hline 1 & 5 & 2 & 2 & \\
\hline 1 & 6 & 2 & 3 & \\
\hline 1 & 7 & 2 & 4 & \\
\hline 1 & 8 & 2 & 2.5 & \\
\hline
\end{tabular}

Criterion 2: (D IL\%) $>50$ and $(\mathrm{N} \mathrm{IL} \%)<50$

\begin{tabular}{|c|c|c|c|c|c|}
\hline 2 & 9 & 1 & 2.5 & 2.4 & 69.3 \\
\hline 2 & 10 & 1 & 2.5 & 3.1 & 64.7 \\
\hline 2 & 11 & 1 & 2.5 & 2.6 & 71.3 \\
\hline 2 & 12 & 1 & 2.5 & 2.6 & 63.5 \\
\hline 2 & 13 & 2 & 0.8 & 1.2 & 78.3 \\
\hline 2 & 14 & 2 & 5 & 4.8 & 58.3 \\
\hline 2 & 15 & 2 & 2.5 & 2.4 & 66.5 \\
\hline 2 & 16 & 2 & 2 & 2.1 & 78.6 \\
\hline \multicolumn{6}{|c|}{ Criterion 3: (D IL\%) minus (N IL\%) >33 } \\
\hline 3 & 17 & 1 & 2 & 2.7 & 72.8 \\
\hline 3 & 18 & 1 & 3 & 3.3 & 59.2 \\
\hline 3 & 19 & 1 & 4 & 3.8 & 75.6 \\
\hline 3 & 20 & 1 & 2 & 2.3 & 70.5 \\
\hline 3 & 21 & 2 & 2 & 2.7 & 73.2 \\
\hline 3 & 22 & 2 & 2 & 2.1 & 68.8 \\
\hline 3 & 23 & 2 & 1.25 & 1.9 & 83.5 \\
\hline 3 & 24 & 2 & 2.5 & 2.6 & 65.6 \\
\hline \multicolumn{3}{|c|}{ Criterion 1 average } & $3.69 * * * *$ & $4.27 *, * *$ & $80.8^{* * * *}$ \\
\hline \multicolumn{3}{|c|}{ Criterion 2 average } & 2.54 & 2.65 & 68.8 \\
\hline \multicolumn{3}{|c|}{ Criterion 3 average } & 2.34 & 2.67 & 71.2 \\
\hline \multicolumn{3}{|c|}{ Squad 1 average } & $3.25 * * *$ & 3.60 & 72.5 \\
\hline \multicolumn{3}{|c|}{ Squad 2 average } & 2.46 & 2.78 & 74.7 \\
\hline
\end{tabular}

${ }^{*} P<0.05$ versus crit $2 ; * * P<0.05$ versus crit $3 ; * * * P<0.10$ versus squad 2

criterion explicitly required a higher level of accuracy than criteria 2 or 3 . However, we expected that most differences between criteria and between squads would express themselves as differences in dosage rather than as differences in accuracy since elevated accuracy will soon lead to a reduced training dose. In agreement with this expectation, differences in criterion stringency resulted in significant differences in LD dose and in average dosage, as reported in Table 1. Also, the apparent lever-choice copying by animals in squad 2 resulted in a significant reduction in LD in squad 2 and a nonsignificant trend in the same direction in average dosage.

Within subgroups, individual differences in LD and average dosage commonly varied by $2: 1$ or more. In human subjects, Perkins et al. (2001) reported a range between subjects approaching 1,000:1 in the threshold-detectable dosage of nicotine. The reasons for such large individual differences are not known, but they necessitate the use of a moderately large number of subjects if an accurate estimate of threshold is desired.

One goal of an experiment like this reasonably might be to obtain an LD that is as close as possible to the absolute threshold. A bias toward the $\mathrm{N}$ lever is commonly observed during D versus $\mathrm{N}$ DD training with depressant drugs. It may have been accentuated in the present experiment because reductions in dosage repeatedly created a situation in which the current training dose was similar to the ED50 dose established during training three or four blocks previously. Because bias occurs, criteria that allow bias in lever choice (e.g., criterion \# 3) will be more permissive than criteria that do not. Previous titration experiments have used criteria that allowed bias (e.g., Colpaert et al. 1980a; Overton 1979, Zenick and Goldsmith 1981), and the present results suggest that such criteria will provide estimates closer to the absolute threshold than criteria that do not allow bias.

The "fixations" that occurred after threshold was reached comprise a difficulty with the titration procedure as it did not, in all rats, work as anticipated. In this study, five out of 24 rats showed fixations. We were able to estimate the LD of those rats on the basis of performance during their first approach to threshold. Nonetheless, these determinations may be less reliable than those obtained from rats that titrated well and repeatedly approached threshold.

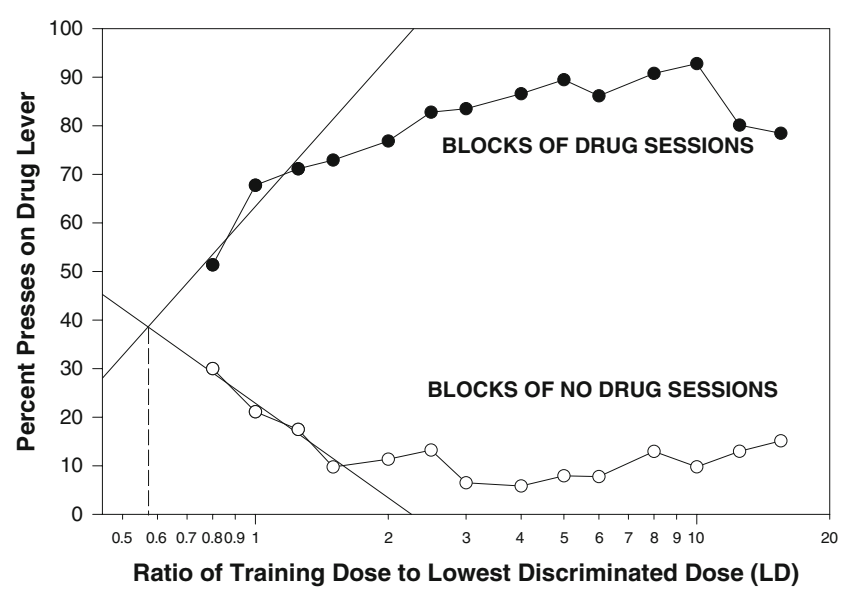

Fig. 2 Accuracy versus dosage. $Y$-axis is average percent presses on the drug lever during the initial links of drug sessions (solid circles) and no-drug sessions (open circles) averaged across the blocks when each dose was used and across rats. $X$-axis is relative training dosage (actual dose divided by the rat's lowest discriminated $(L D)$ dosage. Data points show averages across all rats. The two regression lines were fitted to the data points for the three lowest relative doses to provide one estimate of where absolute threshold might occur. They intersect at a relative dose of 0.57 which corresponds to an average dose of $1.5 \mathrm{mg} / \mathrm{kg}$ 
Finally, the procedures of this experiment do not provide an adequately reliable estimate of the absolute threshold at which drug effects totally cease to acquire discriminative control. Extrapolation, like that in Fig. 2, provides a risky basis for estimating absolute threshold. Different training paradigms that provide data showing the performance of rats at dosages both above and below absolute threshold might provide a more reliable estimate than the one obtained here.

Summary With three different criteria, the experiment determined the lowest dosage of phenobarbital that would produce a criterion level of discriminative control in a drug discrimination paradigm. The results indicate that criteria that allow bias result in lower thresholds for discrimination (LDs) than criteria that do not and that permissive criteria yield lower LDs than more stringent criteria.

The significance of the present results derives from the fact that some theoretically interesting questions only can be answered after trustworthy procedures are available for finding thresholds. Although the present study has not completely defined such a procedure, it takes us a few steps toward that goal.

Acknowledgments This work was supported by grants DA02403 and DA04725 from the National Institute on Drug Abuse and by grant AA09358 from the National Institute on Alcohol Abuse and Alcoholism.

\section{References}

Blough D, Blough P (1977) Animal psychophysics. In: Honig WK, Staddon JER (eds) Handbook of operant behavior. Prentice Hall, Englewood Cliffs, pp 514-539

Colpaert FC, Niemegeers CJE, Janssen PAJ (1977) Differential haloperidol effect on two indices of fentanyl-saline discrimination. Psychopharmacology 53:169-173

Colpaert FC, Niemegeers CJE, Janssen PAJ (1980a) Factors regulating drug cue sensitivity: limits of discriminability and the role of a progressively decreasing training dose in fentanyl-saline discrimination. J Pharm Exp Therap 212:474-480

Colpaert FC, Niemegeers CJE, Janssen PAJ (1980b) Factors regulating drug cue sensitivity: the effect of training dose in fentanylsaline discrimination. Neuropharmacology 19:705-713

Extance K, Goudie AJ (1981) Inter-animal olfactory cues in operant drug discrimination procedures in rats. Psychopharmacology 73:363-371

Overton DA (1979) Drug discrimination training with progressively lowered doses. Science 205:720-721

Overton DA (1982) Comparison of the degree of discriminability of various drugs using the T-maze drug discrimination paradigm. Psychopharmacology 76:385-395

Perkins KA, Fonte C, Sanders M, Meeker J, Wilson A (2001) Threshold doses for nicotine discrimination in smokers and nonsmokers. Psychopharmacology 155:163-170

Preston KL, Bigelow GE (1998) Opioid discrimination in humans: discriminative and subjective effects of progressively lower training dose. Behav Pharm 9:533-543

Tatham TA, Zurn KR (1989) The MED-PC experimental apparatus programming system. Behav Res Meth Instrum Comput 21:294302

Zenick H, Goldsmith M (1981) Drug discrimination learning in leadexposed rats. Science 212:569-571 